\title{
Genome Sequencing Revealed Chromium and Other Heavy Metal Resistance Genes in E. cloacae B2-Dha
}

\author{
Aminur $\mathbf{R}^{1,2 *}$, Björn $\mathrm{O}^{1}$, Jana $\mathbf{J}^{\mathbf{2}}$, Neelu $\mathrm{NN}^{3}$, Sibdas $\mathbf{G}^{4}$ and Abul $\mathbf{M}^{\mathbf{1}}$ \\ ${ }^{1}$ Systems Biology Research Center, School of Bioscience, University of Skövde, Skövde, Sweden \\ ${ }^{2}$ The Life Science Center, School of Science and Technology, Örebro University, SE-701 82 Örebro, Sweden \\ ${ }^{3}$ Microbial Diversity Research Centre, Dr. D.Y. Patil Biotechnology and Bioinformatics Institute, Dr. D. Y. Patil Vidyapeeth, Tathawade, Pune-411033, India \\ ${ }^{4}$ School of Arts and Science, lona College, New Rochelle, NY 10801, USA
}

\begin{abstract}
The previously described chromium resistant bacterium, Enterobacter cloacae B2-DHA, was isolated from leather manufacturing tannery landfill in Bangladesh. Here we report the entire genome sequence of this bacterium containing chromium and other heavy metal resistance genes. The genome size and the number of genes, determined by massive parallel sequencing and comparative analysis with other known Enterobacter genomes, are predicted to be $4.22 \mathrm{Mb}$ and 3958 , respectively. Nearly 160 of these genes were found to be involved in binding, transport, and catabolism of ions as well as efflux of inorganic and organic compounds. Specifically, the presence of two chromium resistance genes, chrR and chrA was verified by polymerase chain reaction. The outcome of this research highlights the significance of this bacterium in bioremediation of chromium and other toxic metals from the contaminated sources.
\end{abstract}

Keywords: Bioremediation; Toxic metals; Enterobacter cloacae; Genome sequencing; De novo assembly; Gene annotation

\section{Introduction}

The global urbanization and industrialization creates increasing levels of pollution including toxic heavy metal contamination [1]. In particular, chromium toxicity is generated through widespread anthropogenic activity via leather processing, steel production, wood preservation, chromium/electroplating, metal processing, alloy formation, textiles, ceramics and thermonuclear weapons manufacturing, and together with agronomic practices such as the use of organic biomass (sewage sludge or fertilizers), which continues to be a major threat to the environment [2-6]. Furthermore, chromium exerts damage directly on human health through toxic and mutagenic effects causing severe DNA damage [7]. However, chromium has multiple effects on bacteria including competitive inhibition of sulphate transport, DNA mutagenesis and protein damage [8]. Microorganisms have developed various mechanisms to survive chromium toxicity: (i) transmembrane efflux of chromate (ii) the ChrR transport system (iii) the reduction of chromate (iv) protection against oxidative stress and (v) DNA repair systems [3,9-15]. In addition, chromate resistance is attributed to the functions of a series of chromosomal or plasmid encoded genes, including the chromium resistance (chr) operon comprising of either chrBAC or chrBACF in bacteria $[9,16,17]$. The ChrA protein, a member of the CHR superfamily of transporters appears to be active in chromate efflux driven by the membrane potential, whereas the $c h r B$ gene encodes for a membrane bound protein necessary for the regulation of chromate resistance [18-20]. The $\operatorname{chr} C$ gene encodes a protein almost similar to iron-containing superoxide dismutase, while the $c h r E$ gene encodes a protein resembling a rhodanese type enzyme in Orthrobacterium tritici 5 bvI1 [20]. The $c h r F$ gene likely encodes a repressor of chromatedependent induction, whereas the ChrR protein catalyzes one-electron shuttle followed by a two-electron transfer to $\mathrm{Cr}^{6+}[21]$

Previously, we have characterized E. cloacae B2-DHA, a soilborne bacterium, that can survive and grow on medium containing up to $5.5 \mathrm{mM}$ chromate. By using inductively coupled plasma atomic emission spectroscopy (ICP-AES) we have shown that after $120 \mathrm{~h}$ of exposure to $100 \mu \mathrm{g} / \mathrm{mL}$ chromium the B2-DHA cells can accumulate $320 \mu \mathrm{g}$ of chromium per gram dry weight of bacterial biomass thus the concentration of chromium in the cell free growth medium is decreased from $100 \mu \mathrm{g} / \mathrm{mL}$ to $19 \mu \mathrm{g} / \mathrm{mL}$ (81\%) [6]. In addition, B2-DHA, can grow on medium containing sodium arsenate, ferric chloride, manganese chloride, zinc chloride, nickel chloride and silver nitrate. However, the mechanisms by which this chromium-adapted B2-DHA survives were not elucidated. Thus, the present study was aimed at demonstrating whether the strain B2-DHA harbored genes that were responsible for chromium and other metal resistance. In this study, we have performed massive parallel genome sequencing of E. cloacae B2-DHA to investigate the metal responsive genes. All the genes involved in metal binding activity and reduction of metal by the E. cloacae B2-DHA strain were predicted by Rapid Annotations using Subsystems Technology, RAST and/or Blast2GO [22,23]. Furthermore, we have conducted comparative genome analyses of $E$. cloacae B2-DHA with other known Enterobacter genome sequences and characterized the genetic rearrangement among the various lineages to understand the evolutionary processes involved in shaping the genomes.

\section{Materials and Methods \\ Extraction of genomic DNA}

Genomic DNA was extracted from E. cloacae B2-DHA using DNeasy Blood \& Tissue Kit (Qiagen, Cat No 69506) according to manufacturer's instructions with some modifications. The bacteria were cultured in Luria Bertani (LB) medium and pellets were collected from $1.0 \mathrm{ml}$ of bacterial cultures by centrifugation at $8000 \mathrm{rpm}$ for $10 \mathrm{~min}$, the

*Corresponding author: Aminur R, Department of Molecular Biology, Systems Biology Research Center, School of Bioscience, University of Skövde, Sweden, Tel: +46-500 448679; +46-7389 81928; E-mail: aminur.rahman@his.se

Received August 28, 2017; Accepted September 18, 2017; Published September 25, 2017

Citation: Aminur R, Björn O, Jana J, Neelu NN, Sibdas G, et al. (2017) Genome Sequencing Revealed Chromium and Other Heavy Metal Resistance Genes in E cloacae B2-Dha. J Microb Biochem Technol 9:191-199. doi: 10.4172/1948 5948.1000365

Copyright: (c) 2017 Aminur R, et al. This is an open-access article distributed unde the terms of the Creative Commons Attribution License, which permits unrestricted use, distribution, and reproduction in any medium, provided the original author and source are credited. 
pellets were resuspended in TE buffer $(10 \mathrm{mM}$ Tris- $\mathrm{HCl}, 1 \mathrm{mM}$ EDTA [pH 8.0]) containing RNase $(50 \mathrm{mg} / \mathrm{ml})$ and lysozyme $(50 \mathrm{mg} / \mathrm{ml})$ and incubated at $37^{\circ} \mathrm{C}$ for $2 \mathrm{~h}$ instead of using ATL (a tissue lysis buffer). The purity and concentration of the extracted DNA were measured using the Nanodrop ND-1000 Spectrophotometer (Saveen Werner, USA). The DNA sample exhibiting a clear band in agarose gel electrophoresis was selected for sequencing of the whole genome.

\section{Genome sequencing}

The entire genome sequencing of $E$. cloacae B2-DHA was assisted by the Otogenetics Corporation (GA, USA) as follows: (i) Purified 0.5$1.0 \mu \mathrm{g}$ of genomic DNA sample was clipped into smaller fragments with a Covaris E210 ultrasonicator; (ii) the library of genomic DNA was prepared according to standard protocol of the NEB library preparation kit (New England Biolabs) for the Illumina sequencer with a single sequencing index; (iii) the sequencing was accomplished with the Illumina HiSeq2500 PE106 (106 bp paired-end) read format; (iv) properly paired reads ( $\geq 30 \mathrm{bp}$ ) were separated from the corrected read pool and the remaining singleton reads were combined as singleend reads; and (v) both of the single-end reads and corrected pairedend reads were used in the subsequent de novo assembly as described previously [24].

\section{De novo assembly}

The de novo assembly started with Illumina 106 bp paired-end reads of genomic DNA with an insert length of $300 \mathrm{bp}$ and the read quality was measured with FastQC, version 1.10.1 [25]. Adapter and quality trimming on raw reads were conducted with cutAdapt and K-mer error correction was performed on the adapter-free reads using Quake, version $0.3 .5[26,27]$. The paired reads were extracted from the corrected read pool and the remaining singleton reads were listed as single-end reads. Both corrected paired-end and single-end reads were used in the $\mathrm{k}$-mer-based de novo assembly. SOAPDenovo, version 2.04 was utilized to perform de novo assembly optimization with the error corrected reads [28]. A wide range of K-mers (29-99) were used to identify the scaffold sequences with the largest N50. The optimal scaffold sequences were further subjected to gap closing by utilizing the corrected pairedend reads, and the resulting scaffolds of length $\geq 300 \mathrm{bp}$ were chosen as the final assembly. The largest N50 of $492,970 \mathrm{bp}$ was produced at the k-mer 97. All the scaffolds were ordered by finding the location of the best Blastn hit for each scaffold on the reference genome E. cloacae ECNIH2 [NCBI accession number CP008823]. A total of 13 scaffolds were used to order the contigs from a draft genome by comparison to a reference genome performed by following the Mauve Contigs Mover (http://darlinglab.org/mauve/user-guide/reordering.html).

\section{Comparative analysis with other E-bacter genomes}

The Whole Genome Shotgun project has been deposited at DDBJ/ EMBL/GenBank under the GenBank accession LFJA00000000 [29]. The progressive MAUVE algorithm in the MAUVE genome alignment software, version 2.3.1was used to study genome rearrangements in $E$. cloacae B2-DHA and related bacteria. Furthermore, another nucleotidebased dot plot analysis was performed with the Gepard software to (i) compare the $4.21 \mathrm{Mbp}$ chromosomal scaffolds of E. cloacae B2-DHA with that of $4.85 \mathrm{Mbp}$ chromosomes in E. cloacae ECNIH2, and (ii) investigate the possible genome rearrangements in these strains.

\section{Prediction and annotation of metal responsive genes}

The prediction of all genes in B2-DHA genome was carried out using FGenesB and GeneMark. ARAGORN, version 1.2.36 employed to predict tRNA genes in B2-DHA genome. We have applied Blast2GO pipeline using all translated protein coding sequences resulting from the FGenesB to execute all functional annotation analyses. In Blast2GO, the BlastP option was chosen to find the closest homologs in the nonredundant protein databases (nr), followed by employment of Gene Ontology (GO) annotation terms to each gene [30]. An InterPro scan was then performed through the Blast2GO interface with the InterPro IDs for obtaining integrated annotation results [31]. Annotation of all putative metal responsive genes was manually curated. The assembled genome sequence was annotated with RAST which uses (i) the GLIMMER algorithm to predict protein-coding genes (ii) the tRNAscan-SE to predict tRNA genes [32], (iii) an internal script for identification of rRNA genes and (iv)the RNAmmer prediction server version 1.2, to identify rRNA genes [33]. Furthermore, RAST (i) infers putative function(s) of the protein coding genes based on homology with known protein families in phylogenetic neighbor species, and (ii) detects subsystems represented in the genome, and helps to reconstruct the metabolic networks. RAST results obtained in prediction of protein coding genes were compared with the GeneMark and the FGenesB algorithms. Circular plot of ordered contigs of B2-DHA was generated with DNAPlotter to predict the graphical map of the genome [34].

\section{PCR amplification of chromium-responsive genes}

Primers for the gene $c h r R$ and $c h r A$ were designed by using the Primer3Plus web tool [35]. The two primer pairs, chrR-F/chrR-R (5'-ATGTCTGATACGTTGAAAGTTGTTA-3'/5'-CAGGCCTTCACCCGCTTA-3') and $c h r A-F / c h r A-R\left(5^{\prime}-T G A A A A G C T G T T T A C C C C A C T-\right.$ 3'/5'-TTACAGTGAAGGGTAGTCGGTATAA-3') were selected for the detection of $c h r R$ and $c h r A$ genes, respectively. PCR amplification of chromium-related marker genes was performed using bacterial genomic DNA as a template in a piko thermal cycler (Finzymes) under the following cycling conditions: $5 \mathrm{~min}$ of denaturation at $95^{\circ} \mathrm{C}$, followed by 30 cycles of $1 \mathrm{~min}$ of denaturation at $95^{\circ} \mathrm{C}, 45 \mathrm{~s}$ of annealing at $54.5^{\circ} \mathrm{C}$ and primer extension at $72^{\circ} \mathrm{C}$ for $1 \mathrm{~min}$ of each $\mathrm{Kb}$ product size. All PCR reaction mixtures contained approximately $50 \mathrm{ng}$ DNA templates, $0.2 \mathrm{mM}$ of each deoxyribonucleoside triphosphate, 1X PCR buffer, $0.5 \mathrm{mM}$ of each primer, and $1 \mathrm{U}$ Taq DNA polymerase in a final volume of $50 \mu$. The final extension reaction was conducted at $72^{\circ} \mathrm{C}$ for 15 min. PCR products were purified with a QIAquick PCR Purification Kit (Qiagen, Cat No 28104).

\section{Results}

\section{Sequencing and de novo genome assembly}

Illumina deep sequencing analysis revealed that the genome of B2-DHA consists of $1,756,877,072$ bases containing $16,574,312$ pairs of reads with an overall GC content of 55\%. After quality trimming error correction followed by removal of the TruSeq adaptor sequence, $15,708,650$ read pairs (94.78\%) and 331,106 single end sequences remained for further analysis. Analysis of the raw reads with FastQC showed that the mean scores per base Phred and per sequence Phred were $\geq 36$ and 36 , respectively for all positions. The set of scaffold sequences with maximal N50 (492,970 bp) was detected at k-mer 97. The corresponding scaffold sequences were subjected to gap closure using the corrected paired-end reads and the resulting scaffolds $(\geq$ $24300 \mathrm{bp}$ ) were defined as the final assembly. The genome summary including the nucleotide content and the gene count is posted in Table 1. The scaffolds were ordered by finding the location of the best Blastn hit for each scaffold on the reference genome Enterobacter cloacae ECNIH2. The final assembly of 4,218,945 bp was comprised of 13 scaffolds ranging from 72,208 to $777,700 \mathrm{bp}$. 
Citation: Aminur R, Björn O, Jana J, Neelu NN, Sibdas G, et al. (2017) Genome Sequencing Revealed Chromium and Other Heavy Metal Resistance Genes in E. cloacae B2-Dha. J Microb Biochem Technol 9:191-199. doi: 10.4172/1948-5948.1000365

\section{Comparative genome analysis}

The chromosomal arrangement of E. cloacae B2-DHA was compared to E. cloacae ECNIH2 by employing progressive Mauve from the Mauve software [36] and Gepard dot plot software [37]. While the alignment remained almost identical in chromosomal rearrangement, the progressive Mauve analysis found several inversions in scaffolds of E. cloacae B2-DHA compared to that in E. cloacae ECNIH2 (Figure 1A). The dot plot performed with E. cloacae B2-DHA and E. cloacae

\begin{tabular}{|l|c|c|}
\hline AttributeT & Value & $\%$ of total \\
\hline Genome size (bp) & 4218945 & 100 \\
\hline DNA GC content (bp) & 2353515 & 55 \\
\hline DNA coding region (bp) & 3768779 & 89,33 \\
\hline Number of replicons & 1 & \\
\hline Total scaffolds & 13 & 100 \\
\hline Total genes & 4043 & 100 \\
\hline rRNA genes & 22 & 0,54 \\
\hline tRNA genes & 66 & 1,63 \\
\hline Protein coding genes & 3958 & 97,82 \\
\hline Genes assigned to RAST functional categories & 3954 & 97,79 \\
\hline Genes assigned Gene Ontology terms by Blast2GO & 3159 & 79,87 \\
\hline Largest N50* & 492970 & \\
\hline Largest N90* & 111054 & \\
\hline
\end{tabular}

Table 1: Summary of the genome of B2-DHA with nucleotide content and gene count.
ECNIH2 depicted a similar observation of inversions in scaffolds of $E$. cloacae B2-DHA (Figure 1B). Furthermore, several large segments of high similarity were obtained when most parts of the chromosomes of E. cloacae B2-DHA and E. cloacae ECNIH2 were mapped onto each other (Figure 1B).

\section{Gene predictions}

The genome and the locations of all genes were predicted through RAST server and the results of this prediction are shown via a circular plot in Figure 2. The prediction of rRNA coding genes showed 22 rRNA genes including four LSU, four SSU, eight $16 \mathrm{~S}$ and six $23 \mathrm{~S}$ genes in $E$. cloacae B2-DHA (Figure 2). ARAGORN, version 1.2.36 [38], employed to predict tRNA genes, identified 66 tRNA genes with a GC content ranging from $48.0 \%$ to $67.5 \%$ in E. cloacae $\mathrm{B} 2$-DHA.

RAST analysis using the GLIMMER algorithm predicted a total of 3958 protein coding genes of which 3401 could be annotated by RAST's automated homology analysis procedure and assigned to functional categories (Figure 3) [27]. For confirmation of the number of protein coding genes, the FGenesB and the GeneMark algorithms were also applied, yielding 3955 and 3764 genes, respectively [39,40]. By using RAST, we observed that the strain E. cloacae B2-DHA contained a large number of genes involved in the ion binding, transport, catabolism and efflux of inorganic as well as organic compounds. More specifically, B2-DHA strain contains many specific metal resistance genes, such
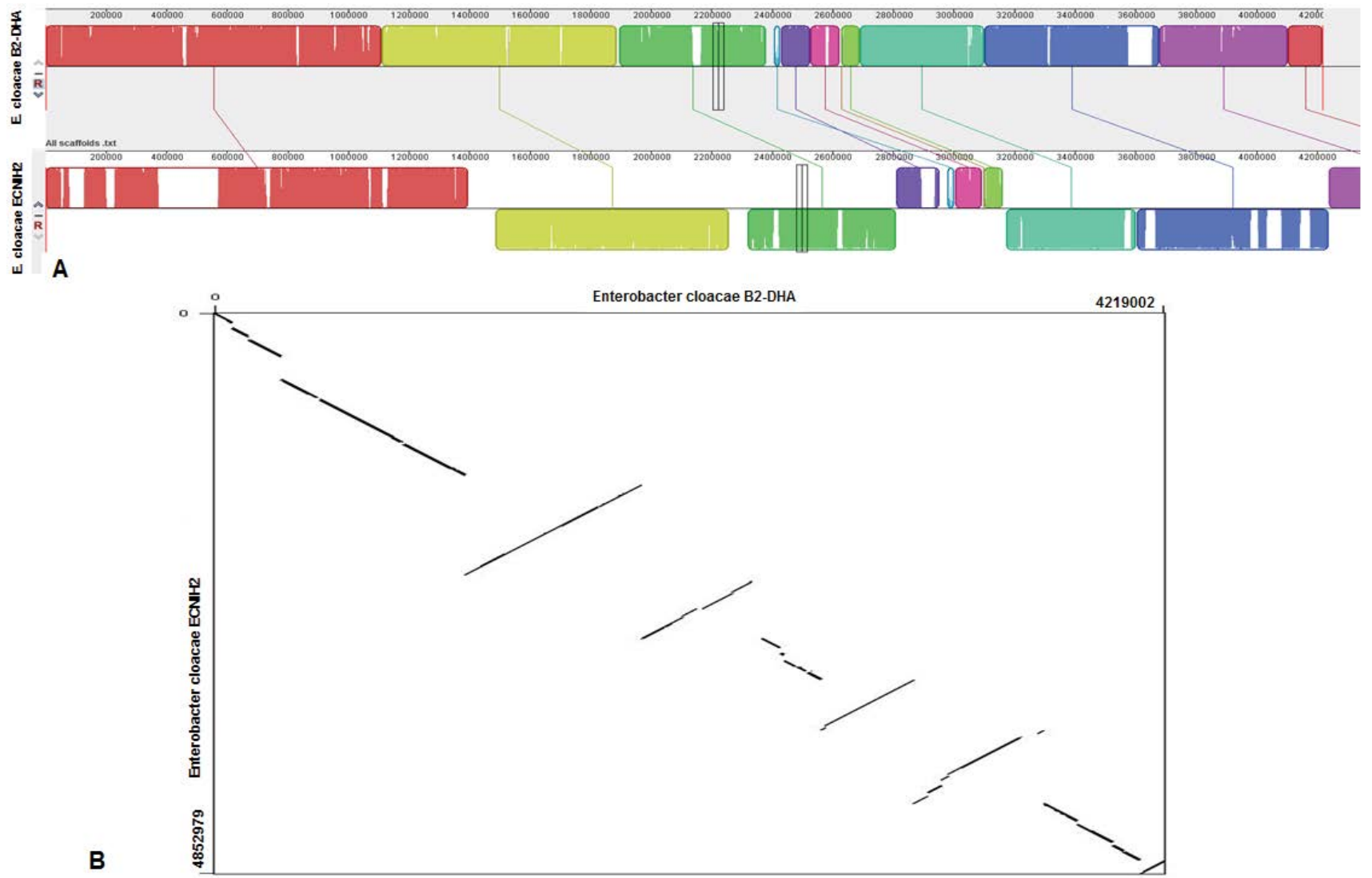

Figure 1: (A) Nucleotide-based alignment of a $4.21 \mathrm{Mbp}$ chromosomal assembly of $E$. cloacae B2-DHA (upper) and $4.85 \mathrm{Mbp}$ chromosomes of $E$. cloacae ECNIH2 (lower). A total of 12 homologous blocks are shown as identically colored regions and linked across the sequences. Regions that are inverted relative to $E$. cloacae (lower). A total of 12 homologous blocks are shown as identically colored regions and linked across the sequences. Regions that are inverted relative to E. cloacae Aligned segments are represented as dots, with regions of conservation appearing as lines. 
Citation: Aminur R, Björn O, Jana J, Neelu NN, Sibdas G, et al. (2017) Genome Sequencing Revealed Chromium and Other Heavy Metal Resistance Genes in E. cloacae B2-Dha. J Microb Biochem Technol 9:191-199. doi: 10.4172/1948-5948.1000365

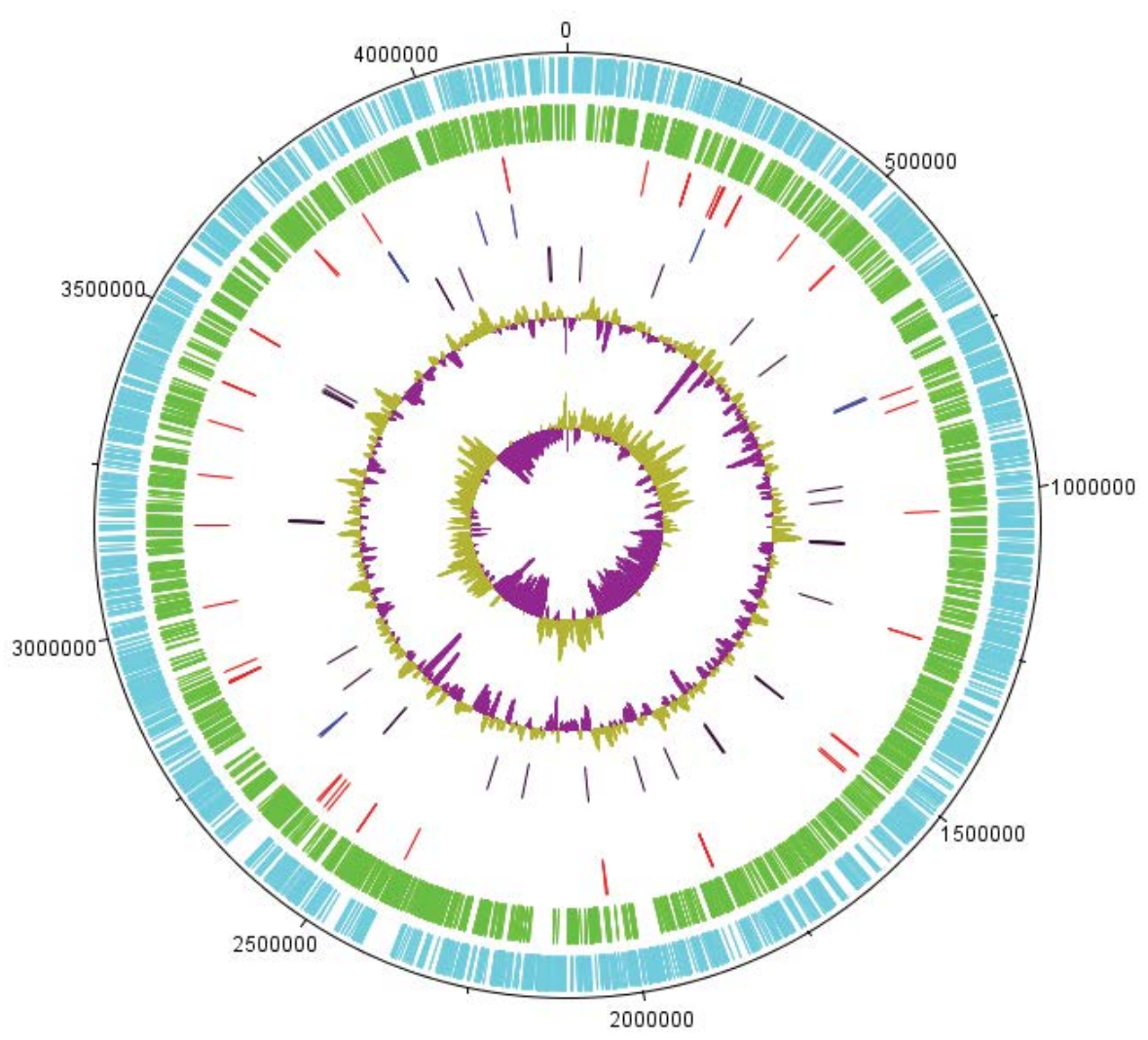

Figure 2: Circular plot of ordered contigs, generated with DNAPlotter. Tracks indicate (from outside inwards) protein coding genes in forward direction (blue) and protein coding genes in reverse direction (green), tRNA genes (red), rRNA genes (dark blue), metal responsive genes (black), GC ratio and GC skew.

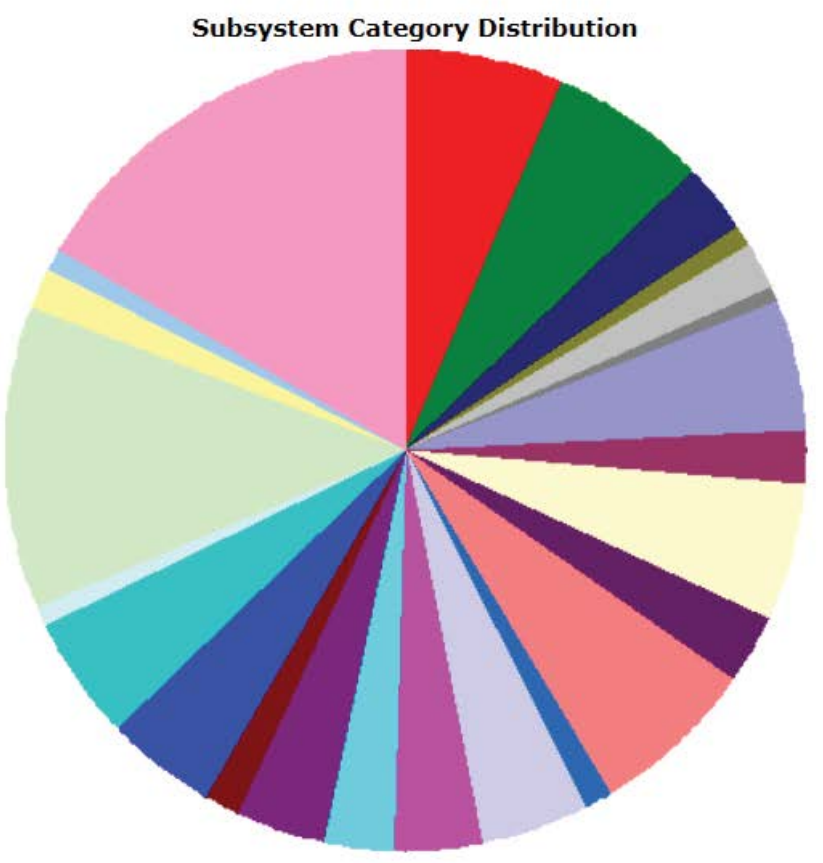

Subsystem Feature Counts

$\boxplus$ Cofactors, Vitamins, Prosthetic Groups, Pigments (225)

$\boxplus$ Cell Wall and Capsule (219)

Virulence, Disease and Defense (93)

$\boxplus$ Potassium metabolism (25)

$\boxplus$ Photosynthesis (0)

Miscellaneous (67)

( Phages, Prophages, Transposable elements, Plasmids (15)

$\boxplus$ Membrane Transport (185)

$\boxplus \square$ Iron acquisition and metabolism (73)

$\boxplus \quad$ RNA Metabolism (193)

$\boxplus \square$ Nucleosides and Nucleotides (93)

$\boxplus$ Protein Metabolism (224)

$\boxplus \square$ Cell Division and Cell Cycle (36)

$\boxplus$ Motility and Chemotaxis (153)

$\boxplus \square$ Regulation and Cell signaling (119)

$\boxplus$ Secondary Metabolism (4)

$\boxplus$ DNA Metabolism (97)

$\boxplus$ Fatty Acids, Lipids, and Isoprenoids (123)

$\boxplus$ Nitrogen Metabolism (46)

$\boxplus$ Dormancy and Sporulation (3)

$\boxplus$ Respiration (156)

$\boxplus$ Stress Response (164)

$\boxplus$ Metabolism of Aromatic Compounds (32)

$\boxplus \quad$ Amino Acids and Derivatives (413)

$\boxplus \quad$ Sulfur Metabolism (60)

$\boxplus$ Phosphorus Metabolism (30)

$\boxplus$ Carbohydrates (553)

Figure 3: RAST analysis of genes connected to subsystems and their distribution in different functional categories. 
Citation: Aminur R, Björn O, Jana J, Neelu NN, Sibdas G, et al. (2017) Genome Sequencing Revealed Chromium and Other Heavy Metal Resistance Genes in E. cloacae B2-Dha. J Microb Biochem Technol 9:191-199. doi: 10.4172/1948-5948.1000365

as arsenic, chromium, cadmium, cobalt, lead and nickel (Table 2). The Blast2GO pipeline analysis also indicated that B2-DHA contains many genes that are directly responsive to toxic metal ions like arsenic, chromium, cadmium, cobalt, lead and nickel (Table 2). Moreover, these analyses revealed that B2-DHA strain also possesses many genes encoding binding and/or transport of calcium, copper, iron, magnesium, potassium and sodium ions as well as several trace elements like manganese, molybdenum and tellurite (Table 3). Also, a large number of zinc ion binding and/or transporter proteins are retained in this strain (Data not shown). Besides zinc the B2-DHA genome contains a total of 104 proteins involved in binding and transport of other metal ions (Data not shown).

\section{Detection of putative chromium resistance genes}

The Blast2GO and RAST analyses detected two chromium reductase genes in B2-DHA. These gene were named as $c h r R$ and $c h r A$ (not to be confused with chromate transporter gene). Presence of these genes in this bacterium was verified by PCR amplification (Figure 4). In addition, a number of other chromate responsive genes were confirmed in B2-DHA. Most of these genes have NAD (P) H dependent oxidoreductase activity (Table 4).

\section{Prediction other proteins}

Several polymyxin resistant proteins such as PmrM, PmrL, PmrJ and ArnC were aslo predicted by RAST and Blast2GO (Table 4). RAST analysis enabled us to detect several multidrug transporter proteins like MdtA, MdtB, MdtC and MdtD in B2-DHA strain (Table 4). This strain also contains universal stress proteins $A, B, C, E$ and $G$, as well as several multiple antibiotic resistance proteins such as MarA, MarB, MarC and MarR (Table 4). Other proteins that catalyze binding and transport

\begin{tabular}{|c|c|c|c|c|}
\hline \multirow[b]{2}{*}{ Start (bp) } & \multirow[b]{2}{*}{ End (bp) } & \multirow[b]{2}{*}{ Predicted function } & \multicolumn{2}{|c|}{ Predicted by } \\
\hline & & & RAST & Blast2GO \\
\hline 36960 & 37526 & Chromate reductase & & $\mathrm{X}$ \\
\hline 242454 & 243404 & Magnesium and cobalt transport protein CorA & $\mathrm{x}$ & $\mathrm{X}$ \\
\hline 495488 & 496147 & ArsR family & $\mathrm{X}$ & \\
\hline 615926 & 616912 & Cobalt, zinc, magnesium ion binding & & $\mathrm{x}$ \\
\hline 964298 & 965137 & Nickel, Cobalt cation transporter activity & & $\mathrm{X}$ \\
\hline 997848 & 1000430 & Copper, lead, cadmium, zinc, mercury transporting ATPase & $\mathrm{X}$ & $\mathrm{X}$ \\
\hline 1100748 & 1099984 & Ferric enterobactin transport protein FepC & $\mathrm{x}$ & $\mathrm{x}$ \\
\hline 1101770 & 1100781 & Ferric enterobactin transport protein FepG & $\mathrm{x}$ & $\mathrm{x}$ \\
\hline 1102774 & 1101770 & Ferric enterobactin transport protein FepD & $\mathrm{x}$ & $\mathrm{x}$ \\
\hline 1105147 & 1104188 & Ferric enterobactin transport protein FepB & $\mathrm{x}$ & $\mathrm{x}$ \\
\hline 1251060 & 1252157 & Chromate reductase & $\mathrm{x}$ & $\mathrm{X}$ \\
\hline 1510555 & 1509272 & Ferrous iron transport peroxidase EfeB & $\mathrm{x}$ & $\mathrm{x}$ \\
\hline 1511686 & 1510559 & Ferrous iron transport periplasmic protein EfeO, & $x$ & \\
\hline 1512560 & 1511727 & Ferrous iron transport permease EfeU & $\mathrm{X}$ & $\mathrm{X}$ \\
\hline 1703726 & 1704046 & Arsenite resistance operon repressor & $\mathrm{x}$ & $\mathrm{x}$ \\
\hline 1704087 & 1705376 & Arsenite efflux pump protein & $\mathrm{X}$ & $\mathrm{X}$ \\
\hline 1705389 & 1705820 & Arsenate reductase & $\mathrm{x}$ & $\mathrm{x}$ \\
\hline 1834407 & 1835345 & Cobalt-zinc-cadmium resistance, Zinc transporter ZitB & $\mathrm{x}$ & $\mathrm{x}$ \\
\hline 1919484 & 1921043 & Magnesium and cobalt efflux protein CorC & $\mathrm{X}$ & \\
\hline 2058555 & 2059283 & Ferric siderophore transport protein TonB & $\mathrm{X}$ & \\
\hline 2216754 & 2216455 & Transcriptional regulator, ArsR family & $\mathrm{X}$ & \\
\hline 2304506 & 2303766 & Cobalt-zinc-cadmium resistance & $\mathrm{x}$ & \\
\hline 2591739 & 2592824 & Cobalt-zinc-cadmium resistance & $\mathrm{X}$ & \\
\hline 2592824 & 2595886 & Cobalt-zinc-cadmium resistance protein CzcA & $\mathrm{X}$ & \\
\hline 2735411 & 2736391 & Nickel, Cobalt cation transporter activity & & $\mathrm{x}$ \\
\hline 2810083 & 2809727 & Arsenate reductase & $\mathrm{x}$ & $\mathrm{x}$ \\
\hline 3168971 & 3169588 & Nickel cation binding & & $\mathrm{x}$ \\
\hline 3169598 & 3170242 & Nickel cation binding & & $\mathrm{x}$ \\
\hline 3170821 & 3171285 & Nickel cation binding & & $x$ \\
\hline 3171295 & 3172998 & Nickel cation binding & & $\mathrm{x}$ \\
\hline 3173316 & 3173618 & Nickel cation binding & & $\mathrm{x}$ \\
\hline 3173629 & 3174456 & Nickel cation binding & & $\mathrm{x}$ \\
\hline 3170811 & 3170272 & Transport of Nickel and Cobalt, Urea decomposition & $\mathrm{x}$ & \\
\hline 3500732 & 3499869 & Nickel incorporation-associated protein HypB & $\mathrm{X}$ & $\mathrm{X}$ \\
\hline 3505195 & 3506904 & Nickel cation binding & & $\mathrm{X}$ \\
\hline 3501086 & 3500736 & Nickel incorporation protein HypA & $\mathrm{x}$ & $\mathrm{X}$ \\
\hline 3516192 & 3517214 & Nickel/cobalt transporter & $\mathrm{X}$ & $\mathrm{X}$ \\
\hline 3892272 & 3892499 & Ferrous iron transport protein $\mathrm{A}$ & $\mathrm{X}$ & $\mathrm{X}$ \\
\hline 3892530 & 3894848 & Ferrous iron transport protein B & $\mathrm{X}$ & \\
\hline 3951655 & 3953826 & Copper, lead, cadmium, zinc, mercury transporting ATPase & $\mathrm{X}$ & $\mathrm{x}$ \\
\hline 4172744 & 4173628 & Cobalt-zinc-cadmium resistance protein & $\mathrm{x}$ & $\mathrm{x}$ \\
\hline 4176907 & 4178211 & Arsenic efflux pump protein & $\mathrm{x}$ & \\
\hline
\end{tabular}

Table 2: Heavy metals responsive proteins in B2-DHA predicted by RAST and/or Blast2GO. 
Citation: Aminur R, Björn O, Jana J, Neelu NN, Sibdas G, et al. (2017) Genome Sequencing Revealed Chromium and Other Heavy Metal Resistance Genes in E. cloacae B2-Dha. J Microb Biochem Technol 9:191-199. doi: 10.4172/1948-5948.1000365

\begin{tabular}{|c|c|c|c|}
\hline Gene & Start & End & Term \\
\hline Gene 802 & 858022 & 859119 & manganese ion binding \\
\hline Gene 196 & 202800 & 204344 & manganese ion binding \\
\hline Gene 209 & 215336 & 216379 & manganese ion binding \\
\hline Gene 271 & 277278 & 279605 & molybdenum ion binding \\
\hline Gene 597 & 628652 & 630154 & manganese ion binding \\
\hline Gene 626 & 664374 & 665294 & manganese ion binding \\
\hline Gene 720 & 771440 & 772036 & manganese ion binding \\
\hline Gene 861 & 919680 & 921458 & manganese ion binding \\
\hline Gene 1016 & 1088133 & 1089044 & Manganese transporter protein SitA \\
\hline Gene 1017 & 1089047 & 1089853 & Manganese transporter protein SitB \\
\hline Gene 1018 & 1089850 & 1090701 & Manganese transporter protein SitC \\
\hline Gene 1019 & 1090695 & 1091534 & Manganese transporter protein SitD \\
\hline Gene 1049 & 1125413 & 1124667 & Molybdenum transport protein ModB \\
\hline Gene 1071 & 1148010 & 1150448 & molybdenum ion binding \\
\hline Gene 1223 & 1294800 & 1296950 & molybdenum ion binding \\
\hline Gene 1504 & 1570809 & 1571876 & molybdenum ion binding \\
\hline Gene 1548 & 1623197 & 1625641 & molybdenum ion binding \\
\hline Gene 1610 & 1694840 & 1695742 & manganese ion binding \\
\hline Gene 1725 & 1822796 & 1821738 & Molybdenum transport protein ModC \\
\hline Gene 1726 & 1823488 & 1822796 & Molybdenum transport protein ModB \\
\hline Gene 1727 & 1824261 & 1823485 & Molybdenum-binding protein ModA \\
\hline Gene 1729 & 1824720 & 1825508 & molybdate ion transport \\
\hline Gene 1756 & 1853924 & 1855090 & manganese ion binding \\
\hline Gene 1818 & 1924327 & 1924905 & manganese ion binding \\
\hline Gene 1903 & 2021204 & 2023633 & molybdenum ion binding \\
\hline Gene 1908 & 2029753 & 2033496 & molybdenum ion binding \\
\hline Gene 2091 & 2224133 & 2227873 & molybdenum ion binding \\
\hline Gene 2098 & 2235200 & 2237611 & molybdenum ion binding \\
\hline Gene 2209 & 2348836 & 2349429 & Tellurite resistance protein TehB \\
\hline Gene 2210 & 2349429 & 2350424 & Tellurite resistance protein TehA \\
\hline Gene 2610 & 2758489 & 2760867 & molybdenum ion binding \\
\hline Gene 2650 & 2805061 & 2806479 & manganese ion binding \\
\hline Gene 2682 & 2845329 & 2847608 & manganese ion binding \\
\hline Gene 2713 & 2876204 & 2877379 & Manganese transport protein $\mathrm{MntH}$ \\
\hline Gene 2785 & 2950966 & 2953689 & molybdenum ion binding \\
\hline Gene 3085 & 3280624 & 3281571 & manganese ion binding \\
\hline Gene 3130 & 3326886 & 3328205 & manganese ion binding \\
\hline Gene 3151 & 3347474 & 3349207 & manganese ion binding \\
\hline Gene 3540 & 3756054 & 3757565 & manganese ion binding \\
\hline Gene 3885 & 4139417 & 4141831 & molybdenum ion binding \\
\hline Gene 3886 & 4141880 & 4142467 & molybdenum ion binding \\
\hline
\end{tabular}

Table 3: Manganese, molybdenum and tellurite resistant proteins in B2-DHA predicted by RAST and/or Blast2GO.

\begin{tabular}{|l|l|l|l|}
\hline Seq. Name & Start & End & Predicted function \\
\hline Gene- 207 & 213103 & 213432 & Thioredoxin \\
\hline Gene- 343 & 355848 & 356153 & Cytochrome-c oxidase activity \\
\hline Gene- 488 & 512342 & 513904 & $\begin{array}{l}\text { Oxidoreductase activity, reduced flavin or } \\
\text { flavoprotein }\end{array}$ \\
\hline Gene- 650 & 692191 & 693615 & Dihydrolipoamide dehydrogenase \\
\hline Gene- 1057 & 1135310 & 1134879 & Universal stress protein G \\
\hline Gene- 1121 & 1198337 & 1199287 & Universal stress protein E \\
\hline Gene- 1150 & 1228221 & 1230242 & NAD(P)H dependent oxidoreductase activity \\
\hline Gene- 1554 & 1634190 & 1635158 & Thioredoxin reductase \\
\hline Gene- 2184 & 2325505 & 2324840 & Multiple antibiotic resistance protein MarC \\
\hline Gene- 2185 & 2325818 & 2326195 & Multiple antibiotic resistance protein MarR \\
\hline Gene- 2186 & 2326216 & 2326596 & Multiple antibiotic resistance protein MarA \\
\hline Gene- 2187 & 2326629 & 2326844 & Multiple antibiotic resistance protein MarB \\
\hline Gene- 2364 & 2487111 & 2487539 & Universal stress protein C \\
\hline Gene- 2436 & 2553300 & 2556080 & NADH: flavin oxidoreductase \\
\hline
\end{tabular}

\begin{tabular}{|l|l|l|l|}
\hline Gene- 2463 & 2591265 & 2590834 & Universal stress protein G
\end{tabular} \begin{tabular}{|l|l|l|l|l|}
\hline Gene- 2531 & 2669613 & 2672735 & Multidrug resistance MdtB \\
\hline
\end{tabular} \begin{tabular}{|l|l|l|l|}
\hline Gene- 2533 & 2672736 & 2675813 & Multidrug resistance MdtC
\end{tabular} \begin{tabular}{|l|l|l|l|l|}
\hline Gene- 2534 & 2675814 & 2677229 & Multidrug resistance MdtD \\
\hline
\end{tabular} \begin{tabular}{|l|l|l|l|l|}
\hline Gene- 3334 & 3544616 & 3543069 & Multidrug resistance MdtB \\
\hline
\end{tabular} \begin{tabular}{|l|l|l|l|}
\hline Gene- 3335 & 3545805 & 3544633 & Multidrug resistance MdtA
\end{tabular} \begin{tabular}{|l|l|l|l|l}
\hline Gene- 3582 & 3809416 & 3810390 & Quinone oxidoreductase \\
\hline
\end{tabular} \begin{tabular}{|l|l|l|l|l|}
\hline Gene- 3638 & 3860171 & 3862714 & Nitrite reductases \\
\hline
\end{tabular} \begin{tabular}{|l|l|l|l|l|}
\hline Gene- 3746 & 3974324 & 3973941 & Polymyxin resistance protein PmrM
\end{tabular} \begin{tabular}{|l|l|l|l|}
\hline Gene- 3747 & 3974644 & 3974321 & Polymyxin resistance protein PmrL,
\end{tabular} \begin{tabular}{|l|l|l|l|l|}
\hline Gene- 3749 & 3977186 & 3976284 & Polymyxin resistance protein PmrJ
\end{tabular} \begin{tabular}{|l|l|l|l|}
\hline Gene- 3751 & 3980145 & 3979162 & Polymyxin resistance protein ArnC
\end{tabular} \begin{tabular}{|l|l|l|l|}
\hline Gene- 3760 & 3989137 & 3988850 & Universal stress protein B \\
\hline
\end{tabular} \begin{tabular}{|l|l|l|l|l|}
\hline Gene- 3761 & 3989469 & 3989906 & Universal stress protein A
\end{tabular}

Table 4: Universal stress proteins, multiple antibiotic resistant proteins, multidrug resistance proteins and polymyxin resistance protein in B2-DHA as predicted by RAST and/or Blast2GO. 


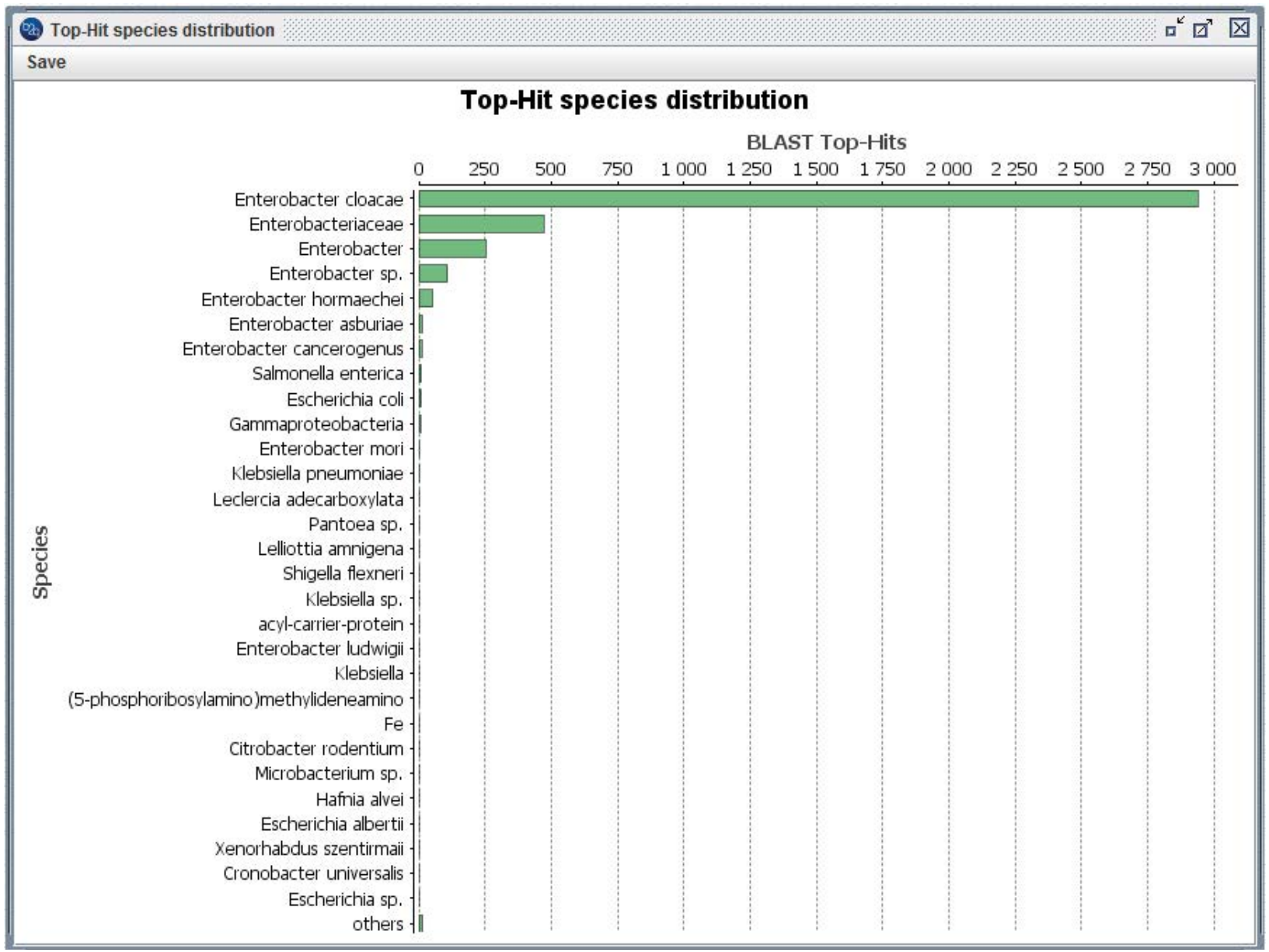

Figure 4: Molecular analysis of chromium responsive genes of B2-DHA and gel electrophoresis. PCR amplification of chrR and chrA genes. L represents 2 log DNA marker, lane 1 and 2 are the amplified fragments of chrR gene in two replicates whereas lane 3 and 4 are the amplified fragments of chrA gene in two replicates.

of the metal ions are metalloendopeptidase, metalloexopeptidase, metallopeptidase, metallocarboxypeptidase and metallochaperone. Some metallocenter assembly proteins such as HypA, HypB, HypC, HypD, HypE and HypF are also present in this strain.

\section{Discussion}

Previously we have reported chromium-resistant bacterial strain E. cloacae B2-DHA isolated from the Hazaribagh tannery areas in Bangladesh [6]. In this paper we report the results of sequencing of the whole-genome of this bacterium. After quality trimming, error correction, and removal of the TruSeq adaptor sequence the genome was de novo assembled resulting an approximate genome length of 4.22 Mbp. Several other Enterobacter strains have been sequenced previously. For example, E. cloacae UW5 had a genome size of 4.9-Mbp and E. cloacae ENHKU01 had 4.72-Mbp [41,42]. Our strain E. cloacae B2-DHA contained a total of 3958 protein coding genes, whereas in E. cloacae ENHKU01 the total number of these genes was 4338 . The results we obtained in B2-DHA are in agreement with those reported by other researchers, although the genome size and number of protein coding genes in $\mathrm{B} 2$-DHA are slightly smaller than those in E. cloacae ENHKU0. The difference in number of protein coding genes in the bacterial strains can be attributed to a common phenomenon. Even in a Gram-positive bacterium, Lysinibacillus sphaericus B1-CDA, the number of protein coding genes analyzed by different web tools was found to be different $[43,44]$. The goal of gene prediction in B2DHA was to catalogue all the genes encoded within its genome. This prediction facilitates understanding of the mechanisms that might be involved in resistance of this bacterium to chromium and other toxic metals. The annotation of the assembled genome, number of tRNA and rRNA in B2-DHA varied from those found in the reference genome of E. cloacae ECNIH2. B2-DHA genome contained 22 rRNA and 66 tRNA genes, whereas in the reference genome ECNIH2 these were 25 and 87, respectively (http://www.ncbi.nlm.nih.gov/nuccore/CP008823). The difference in the number of tRNA genes is a common feature of bacterial and archaeal genomes [45]. These differences could likely be due to the draft status of their B2-DHA genome compared to the reference. However, sometimes annotation systems miss some RNA genes. Furthermore, the bacteria which have the highest number of $16 \mathrm{~S}$ rRNA genes also have the highest number of tRNA genes [46].

Results obtained from RAST and Blast2GO analyses showed that the bacterium contains many metal resistance genes and there is no significant difference in the obtained results between these two methods (Table 2). Genome sequencing also revealed that B2-DHA harbors many other genes conferring resistance of this bacterium to 
polymyxins, multiple drugs and antibiotics. These type genes or their homologues have been identified previously in both Gram-positive and Gram-negative bacteria as well as archaea [47,48]. The proteins encoded by these genes contain many metal-binding residues, which may bind to several metal ions, primarily nickel ions $[49,50]$. Polymyxin resistance proteins are polycationic antimicrobial peptides that serve as antibiotics for the treatment of infectious diseases caused by multidrug-resistant Gram-negative bacteria. Several bacteria such as Serratia sp., Burkholderia sp. and Proteus sp. are naturally resistant to these antibiotics, whereas other bacteria like Pseudomonas aeruginosa, Acinetobacter baumannii and Klebsiella pneumoniae develop resistance to polymyxins through acquired resistance [51]. The B2-DHA strain contains many metalloproteinase or metalloprotease enzymes. The possible explanation for this is that the bacteria often need to protect themselves from adverse environmental stimuli, including exposure to stress factor, cationic antimicrobial peptides, and toxic metals [52]. To survive in these stress conditions bacteria develop various strategies mainly based on alterations of the lipopolysaccharides (LPSs) in their cell walls, which have overall negative charges and are the initial targets of polymyxins [53]. Other strategies may include efflux pumps and capsule formation $[54,55]$. Thus, the strain B2-DHA, isolated from highly chromium contaminated tannery industry area may have developed similar mechanisms to survive under adverse conditions.

We also report that B2-DHA contains 219 genes which are responsive to cell wall and capsule development as well as 164 genes which are involved in stress response (Figure 3). Presence of these genes in this bacterium might be accounted for its morphological changes when exposed to chromium. This type of changes is an advantageous trait for this bacterium and it facilitates accumulation of chromium inside the cells [6]. As described in the results, B2-DHA contains a number of chromate reductase genes and most of these have NAD $(\mathrm{P})$ H-dependent oxidoreductase activity (Table 4). Similar kind of results has been reported previously by [56]. B2-DHA also harbors soluble quinone oxidoreductases that are expected to reduce $\mathrm{Fe}^{3+}$ and $\mathrm{Cr}^{6+}$ and counter oxidative stress [57]. In addition, B2-DHA possesses many other functional genes such as thioredoxin and thioredoxin reductase, dihydrolipoamide dehydrogenase, nitrite reductases, NADH: flavin oxidoreductase, quinones, cytochromes, flavoproteins and proteins with iron sulphur centers (Table 4). These genes are believed to be involved in metal oxidoreductase exhibiting $\mathrm{Cr}^{6+}$ reduction as reported previously [58-60]. The proteins encoded by these genes initially catalyze one-electron shuttle followed by a two-electron transfer to $\mathrm{Cr}^{6+}$ with the formation of intermediate(s) $\mathrm{Cr}^{5+}$ and/or $\mathrm{Cr}^{4+}$ before further reduction to $\mathrm{Cr}^{3+}$ which is a critical process involved in detoxification of chromium inside the cells [21]. Thus bacteria can survive and grow in a chromium contaminated environment. One of many possible ways to increase the effectiveness of chromium bioremediation by using bacteria is to alter the expression of these genes to minimize oxidative stress during chromate reduction. This approach has been proposed by several other researchers $[12,48]$. Previously, we have postulated that the B2-DHA is resistant to chromium and it can decrease chromium content significantly in the contaminated source by accumulating it in the cells [6]. Furthermore, we report that the bacterium can reduce $\mathrm{Cr}^{6+}$ to $\mathrm{Cr}^{3+}$, corroborating the presence of chromium resistance genes $\mathrm{chr} R$ and $c h r A$ as described in this paper.

\section{Conclusion}

In this paper we report the genome sequence and annotation of a chromium resistant bacterium, E. cloacae B2-DHA. Furthermore, bioinformatics analyses revealed that this bacterium harbours two chromium resistance genes, chrA and chrR among many metal resistance and other genes. Our previous findings of chromium accumulation and the recent data on genomics and functionality of the genes in B2-DHA (which is under investigation) will provide insights to establish the mechanism of chromium resistance in this strain. Altogether, our findings can be employed in bioremediation of these toxic metals in polluted environments especially industry effluents. In a long-term perspective, millions of people worldwide, in turn, can avoid many lethal diseases caused by chronic exposure of toxic metal poisoning. Therefore, our discoveries have a great potential through further investigations in contributing to a significant positive impact on the socioeconomic status of the people particularly in the developing world.

\section{Acknowledgement}

This research was supported by a major grant (AKT-2010-018) from the Swedish International Development Cooperation Agency (SIDA), and partly by a small grant from the Nilsson-Ehle (The Royal Physiographic Society in Lund) foundation in Sweden.

\section{References}

1. Rahman A, Nahar N, Nawani NN, Jass J, Desale P, et al. (2014) Isolation of a Lysinibacillus strain B1-CDA showing potentials for arsenic bioremediation. J Environ Sci Health A Tox Hazard Subst Environ Eng 49: 1349-1360.

2. Viti C, Pace A, and Giovannetti L (2003) Characterization of $\mathrm{Cr}(\mathrm{VI})$-resistant bacteria isolated from chromium-contaminated soil by tannery activity. Curr Microbiol 46: 1-5.

3. Chourey K, Thompson MR, Morrell-Falvey J, VerBerkmoes NC, Brown SD, et al. (2006) Global molecular and morphological effects of $24 \mathrm{~h}$ chromium(vi) exposure on Shewanella oneidensis MR-1. Appl Environ Microbiol 72: 63316344.

4. Saha R, Nandi R, Saha B (2011) Sources and toxicity of hexavalent chromium: A review. J Coord Chem 64: 1782-1806.

5. Kamika I, Momba M (2013) Assessing the resistance and bioremediation ability of selected bacterial and protozoan species to heavy metals in metal-rich industrial wastewater. BMC Microbiol 13: 28

6. Rahman A, Nahar N, Nawani NN, Jass J, Hossain K, et al. (2015a) Bioremediation of hexavalent chromium (VI) by a soil borne bacterium, Enterobacter cloacae B2-DHA. J Environ Sci Health A Tox Hazard Subst Environ Eng 50: 1136-1147.

7. Viti C, Marchi E, Decorosi F, Giovannetti L (2014) Molecular mechanisms of $\mathrm{Cr}(\mathrm{VI})$ resistance in bacteria and fungi. FEMS Microbiol Rev 38: 633-659.

8. He M, Li X, Guo L, Miller SJ and Rensing C (2010) Characterization and genomic analysis of chromate resistant and reducing Bacillus cereus strain SJ1. BMC Microbiol. 10: 1-10.

9. Ramirez-Diaz M, Diaz-Perez C, Vargas E, Riveros-Rosas H, Campos-Garcia $\mathrm{J}$, et al. (2008) Mechanisms of bacterial resistance to chromium compounds. Biometals 21: 321-332.

10. Saier MH (2003) Tracing pathways of transport protein evolution. Mol Microbiol 48: $1145-1156$

11. Cervantes C, Campos-Garcia J (2007) Reduction and efflux of chromate by bacteria. In: Nies, D. H., Silver S. (Eds.). Molecular Microbiology of Heavy Metals (ed. Nies DH, Silver S, Springer-Verlag), pp: 407-420.

12. Ackerley DF, Barak Y, Lynch SV, Curtin J, Matin A (2006) Effect of chromate stress on Escherichia coli K-12. J Bacteriol 188: 3371-3381.

13. Brown SD, Thompson MR, Verberkmoes NC, Chourey K, Shah M, et al. (2006) Molecular dynamics of the Shewanella oneidensis response to chromate stress. Mol Cell Proteomics 5: 1054-1071.

14. Henne KL, Nakatsu CH, Thompson DK, Konopka AE (2009) High-leve chromate resistance in Arthrobacter sp. strain FB24 requires previously uncharacterized accessory genes. BMC Microbiol 9: 199

15. Miranda AT, González MV, González EG, Vargas E, Campos-García J, et al. (2005) Involvement of DNA helicases in chromate resistance by Pseudomonas aeruginosa PAO1. Mutat Res 578: 202 - 209. 
Citation: Aminur R, Björn O, Jana J, Neelu NN, Sibdas G, et al. (2017) Genome Sequencing Revealed Chromium and Other Heavy Metal Resistance Genes in E. cloacae B2-Dha. J Microb Biochem Technol 9:191-199. doi: 10.4172/1948-5948.1000365

16. Nies A, Nies DH, Silver S (1990) Nucleotide sequence and expression of a plasmid encoded chromate resistance determinant from Alcaligens eutrophus. J Biol Chem 265: 5648-5653.

17. Branco R, Chung AP, Johnston T, Gurel V, Morais P, et al. (2008) The chromateinducible chrBACF operon from the transposable element TnOtChr confers resistance to chromium(VI) and superoxide. J Bacteriol 190: 6996-7003.

18. Henson MW, Domingo JWS, Kourtev PS, Jensen RV, Dunn JA, et al (2015) Metabolic and genomic analysis elucidates strain-level variation in Microbacterium spp. isolated from chromate contaminated sediment. Peer $J$ 3: e1395.

19. Pimentel BE, Sa'nchez RM, Cervantes C (2002) Effux of chromate by Pseudomonas aeruginosa cells expressing the ChrA protein. FEMS Microbiol Lett 212: 249-254.

20. Morais PV, Branco R,Francisco R (2011) Chromium resistance strategies and toxicity: What makes Ochrobactrum tritici $5 \mathrm{bvl} 1$ a strain highly resistant Biometals 24: 401-410.

21. Cheung KH, Ji-Dong G (2007) Mechanism of hexavalent chromium detoxification by microorganisms and bioremediation application potential: A review. Int Biodeterior Biodegrada 59: 8-15.

22. Aziz RK, Bartels D, Best AA, DeJongh M, Disz T, et al. (2008) The RAST server: Rapid annotations using subsystems technology. BMC Genomics 9: 75.

23. Götz S, García-Gómez JM, Terol J, Williams TD, Nagaraj SH, et al. (2008) High-throughput functional annotation and data mining with the Blast2GO suite. Nucleic Acids Res 36: 3420-3435

24. Rahman A, Nahar N, Jass J, Olsson B, Mandal A (2016a) Complete genome sequence of Lysinibacillus sphaericus B1-CDA, a bacterium that accumulates arsenic. Genome Announc 4: e00999-15.

25. Andrews S (2010) FastQC: A quality control tool for high throughput sequence data.

26. Martin M (2011) Cutadapt removes adapter sequences from high-throughput sequencing reads. EMBnet 17:10-12.

27. Kelley DR, Schatz MC, Salzberg SL (2010) Quake: Quality-aware detection and correction of sequencing errors. Genome Biol 11: R116.

28. Luo R, Liu B, Xie Y, Li Z, Huang W, et al. (2012) SOAPdenovo2: An empirically improved memory-efficient short-read de novo assembler. GigaScience 1: 18.

29. Rahman A, Nahar N, Olsson B, Mandal A (2016b) Complete genome sequence of Enterobacter cloacae B2-DHA, a chromium resistant bacterium. Genome Announc 4: e00483-16.

30. Ashburner M, Ball CA, Blake JA, Botstein D, Butler H, et al. (2000) Gene ontology: Tool for the unification of biology. The Gene Ontology Consortium. Nat Genet 25: 25-29.

31. Zdobnov EM, Apweiler R (2001) InterProScan - an integration platform for the signature-recognition methods in InterPro. Bioinformatics 17: 847-848.

32. Lowe TM, Eddy SR (1997) tRNAscan-SE: A program for improved detection of transfer RNA genes in genomic sequence. Nucleic Acids Res 25: 955-964.

33. Lagesen K, Hallin P, Rødland EA, Stærfeldt HH, Rognes T, et al. (2007) RNAmmer: Consistent and rapid annotation of ribosomal RNA genes. Nucleic Acids Res 35: 3100-3108

34. Carver T, Thomson N, Bleasby A, Berriman M, Parkhill J (2009) DNAPlotter: Circular and linear interactive genome visualization. Bioinformatics (Oxford, England) 25: 119-120.

35. Untergasser A, Nijveen H, Rao X, Bisseling T, Geurts R, (2007) Leunissen: Primer3Plus, an enhanced web interface to Primer3. Nucleic Acids Res 35 : W71-W74.

36. Darling AE, Mau B, Perna NT (2010) ProgressiveMauve: Multiple genome alignment with gene gain, loss and rearrangement. PLoS ONE 5: e11147.

37. Krumsiek J, Arnold R, Rattei T (2007) Gepard: A rapid and sensitive tool for creating dot plots on genome scale. Bioinformatics 23:1026-1028.

38. Laslett D, Canback B (2004) ARAGORN, a program to detect tRNA genes and tmRNA genes in nucleotide sequences. Nucleic Acids Res 32: 11-16.

39. Salzberg SL, Delcher AL, Kasif S, White O (1998) Microbial gene identification using interpolated Markov models. Nucleic acids Res 26: 544-548.
40. Salamov AA, Solovyev VV (2000) Ab initio gene finding in Drosophila genomic DNA. Genome Res 10: 516-522.

41. Borodovsky M, Mclninch J (1993) GeneMark: parallel gene recognition for both DNA strands. Comput Chem 17: 123-133.

42. Coulson TJD, Patten CL (2015) Complete genome sequence of Enterobacter cloacae UW5, a Rhizobacterium capable of high levels of indole-3-acetic acid production. Genome Announc 3: e00843-15.

43. Liu WY, Wong CF, Chung KMK, Jiang JW, Leung FCC (2013) Comparative genome analysis of Enterobacter cloacae. PLoS ONE 8: e74487.

44. Rahman A, Nahar N, Nawani NN, Jass J, Ghosh S, et al. (2015b) Comparative genome analysis of Lysinibacillus B1-CDA, a bacterium that accumulates arsenics. Genomics 106: 384-392.

45. Lee ZMP, Bussema C, Schmidt TM (2009) rrnDB: documenting the number of rRNA and tRNA genes in bacteria and archaea. Nucleic Acids Res 37: D489-D493.

46. Vezzi A, Campanaro S, D’Angelo M, Simonato F, Vitulo N, et al. (2005) Life a depth: Photobacterium profundum genome sequence and expression analysis. Science 307: 1459-1461.

47. Paschos A, Bauer A, Zimmermann A, Zehelein E, Böck A (2002) HypF, carbamoyl phosphate-converting enzyme involved in [NiFe] hydrogenase maturation. J Biol Chem 277: 49945-49951.

48. Rahman A, Nahar N, Nawani NN, Jass J, Ghosh S, et al. (2015c) Data in support of the comparative genome analysis of Lysinibacillus B1-CDA, a bacterium that accumulates arsenics. Data Brief 5: 579-585.

49. Olaitan AO, Morand S, Rolain JM (2014) Mechanisms of polymyxin resistance: Acquired and intrinsic resistance in bacteria. A review article. Front Microbio 5: 1-18.

50. Moffatt JH, Harper M, Harrison P, Hale JD, Vinogradov E, et al. (2010) Colistin resistance in Acinetobacter baumannii is mediated by complete loss of lipopolysaccharide production. Antimicrob Agents Chemother 54: 4971-4977.

51. Campos MA, Vargas MA, Regueiro V, Llompart CM, Alberti S, et al. (2004) Capsule polysaccharide mediates bacterial resistance to antimicrobia peptides. Infect Immun 72: 7107-7114.

52. Padilla E, Llobet E, Domenech-Sanchez A, Martinez-Martinez L, Bengoechea $\mathrm{JA}$, et al. (2010) Klebsiella pneumoniae AcrAB efflux pump contributes to antimicrobial resistance and virulence. Antimicrob Agents Chemother 54: 177-183.

53. Ramírez-Díaz MI, Díaz-Pérez C, Vargas E, Riveros-Rosas H, Campos-García $\mathrm{J}$, et al. (2007) Mechanisms of bacterial resistance to chromium compounds. BioMetals 21: 321-332.

54. McCord JM, Fridovich I (1988) Superoxide dismutase: the first twenty years (1968-1988). Free Radic. Biol Med 5: 363-369.

55. Myers CR, Carstens BP, Antholine WE, Myers JM (2000) Chromium (VI) reductase activity is associated with the cytoplasmic membrane of anaerobically grown Shewanella putrefaciens MR-1. J Appl Microbiol 88: 98-106.

56. Viamajala S, Peyton BM, Apel WA, Petersen JN (2002) Chromate/nitrite interactions in Shewanella oneidensis MR-1: Evidence for multiple hexavalent chromium $[\mathrm{Cr}(\mathrm{VI})]$ reduction mechanisms dependent on physiological growth conditions. Biotechnol Bioeng 78: 770-778.

57. Ackerley DF, Gonzalez CF, Keyhan M, Blake R, Matin A (2004) Mechanism of chromate reduction by the Escherichia coli protein, $\mathrm{Nfs} A$ and the role of different chromate reductases in minimizing oxidative stress during chromate reduction. Environ Microbiol 6: 851-860.

58. Opperman DJ, Van Heerden E (2008) A membrane-associated protein with $\mathrm{Cr}(\mathrm{VI})$-reducing activity from Thermus scotoductus SA-01. FEMS Microbiol Lett 280: 210-218

59. Li X, Krumholz LR (2009) Thioredoxin is involved in $\mathrm{U}(\mathrm{VI})$ and $\mathrm{Cr}(\mathrm{VI})$ reduction in Desulfovibrio desulfuricans G20. J Bacteriol 191: 4924-4933.

60. Bhattacharya P, Barnebey A, Zemla M, Goodwin L, Auer M, et al. (2015) Complete genome sequence of the chromate-reducing bacterium Thermoanaerobacter thermohydrosulfuricus strain BSB-33. Standards in Genomic Sciences 10: 74.

This article was originally published in a special issue, Advances in Microbiology and Biołechnology handled by Editor(s). Dr. Gamil Sayed Gamil Zeedan, , National Research Center, Egypt 